\title{
Non-Contact Micro-Liquid Mixing Method Using Ultrasound*
}

\author{
Hajime KATOU**, Ryo MIYAKE ${ }^{* * *}$ and Takao TERAYAMA ${ }^{* * * *}$
}

\begin{abstract}
A new mixing method for micro-liquid has been developed. In the new method, a radiation pressure of ultrasound is used to stir liquid in a vessel without any physical contact. The developed mixing device based on the method consists of a vessel containing the liquid to be mixed and two ultrasound sources (frequency: $1.6 \mathrm{MHz}$ ) arranged outside the vessel. One of them radiates through the bottom, the other through the side wall of the vessel. The latter sound source is divided into several segments. And the fine position of the sound source corresponding to the level of liquid is adjusted by selecting the appropriate segments. The mixing performance is evaluated according to the difference between the two local transmissibilities of laser beam in the liquid. As a result, we confirmed that by using the mixing device, the liquid $(80-160 \mu \mathrm{L}$ of water and dye) become homogeneous within $1.0 \mathrm{sec}$.
\end{abstract}

Key Words: Mixing, Non-Contact, Ultrasound, Arrayed Sound Source

\section{Introduction}

If chemical analysis could be conducted with lower volumes of sample and reagent, several advantages would emerge. For example, the cost of testing and the amount of waste after testing would be reduced because less reagents and samples would be needed. Also it would reduce the discomfort of patients from whom smaller amount of specimen need to be obtained for diagnosis.

To maintain the accuracy of analysis at reduced sample and reagent volumes, it is important to insure proper mixing. Paddles or screws used in conventional automatic chemical analyzer are immersed directly in the liquid and rotated. Inevitably the mixers end up reducing the sample volume by taking a portion of the liquid; this would be a cause for errors. Another serious problem with conventional mixing method, i.e., cross contamination occurs between liquids in different vessels. To avoid the problem, the paddle or screw needs to be washed after each mixing

* Received 11th August, 2004 (No. 00-0699). Japanese Original: Trans. Jpn. Soc. Mech. Eng., Vol.66, No.652, B (2000), pp.3137-3142 (Received 29th May, 2000)

** Hitachi, Ltd. Mechanical Engineering Research Laboratory, MEMS project, 832-2 Horiguchi, Hitachinaka, Ibaraki 312-0034, Japan.

E-mail: hajime.kato.sx@hitachi.com

*** Hitachi, Ltd. Mechanical Engineering Research Laboratory, MEMS project, 832-2 Horiguchi, Hitachinaka, Ibaraki 312-0034, Japan

**** iTiD Consulting, Ltd., 4-11-10 Nakanoku-nakano, Tokyo 164-0001, Japan process in the conventional analyzers. However, this is not enough at low volumes because residual materials cannot be ignored in such small volumes. If excessive detergent and water are used to remove such pollutants, the amount of waste also increases and consequently the advantages of low-volume chemical analysis would be greatly reduced.

If mixing could be done without physical contacting the liquid, (i.e., without using a paddle or screw) the above problems could be completely solved. The method is called "non-contact mixing" and several non-contact mixing devices have recently been developed ${ }^{(1),(2)}$, which mix liquids with a pressure wave. Both of them require the liquid to be hermetically sealed within a vessel. However, many current automatic chemical analyzers have open vessels containing liquids with a free surface. Therefore, we developed a non-contact mixing method that could be applied to the liquid with the free surface.

In this paper, we discussed our approach for the noncontact mixing method using radiation pressure of ultrasound and proposed a specific device configuration for practical application. In addition, the mixing performance of the device was evaluated using flow visualization technique and the mixing performance was also evaluated.

\section{Non-Contact Mixing Using Ultrasound}

\subsection{Fundamental principle}

As shown in Fig. 1 (a), when ultrasound propagating in a liquid reaches its free surface, an acoustic radiation pressure $^{(3)-(5)}$ appears and expels the free surface towards the air. A photograph of this "push-out" phenomenon is in Fig. 1 (b). The effects of acoustic radiation pressure de- 


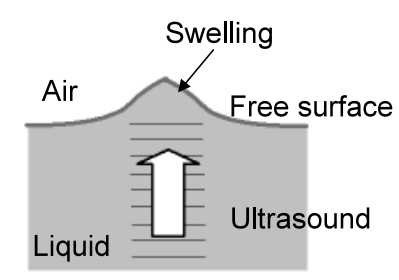

(a) Schematic illustration

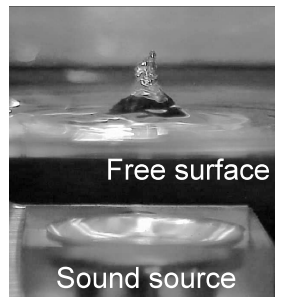

(b) Photograph

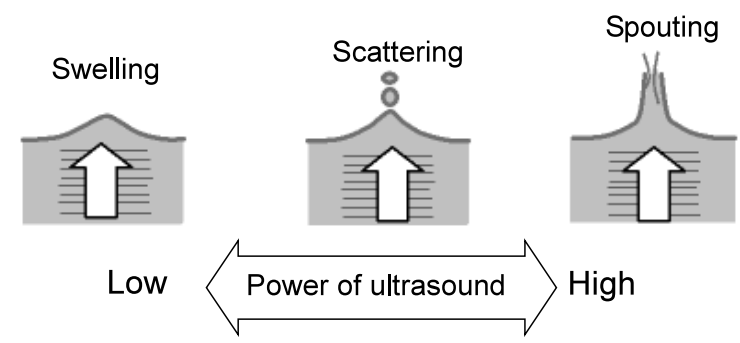

(c) Relationship between swelling and power of ultrasound

Fig. 1 Radiation of ultrasound generated at a free surface

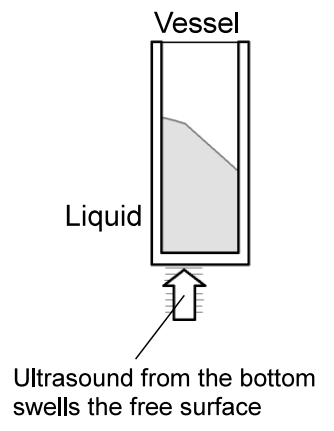

(a) Irradiation from bottom

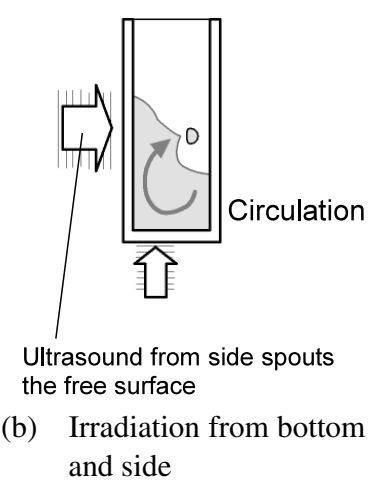

Fig. 2 Schematic diagram of non-contact mixing method

pend on the nature of the medium, as well as the frequency and power of the sound. These effects are easily observable when a peak-to-peak voltage of $30-40 \mathrm{~V}$ is supplied to a piezoelectric element and the sound frequency is on the order of MHz. Figure 1(c) schematically shows how swelling becomes scattering, then spouting, as ultrasound power increases.

We applied this "push-out" phenomenon to be a basis of our non-contact mixing method. The mixing mechanism that we speculated are explained as follows. Figure 2 (a) shows that a weak ultrasound is transmitted upward from the bottom of the vessel (skewed to the left here), thereby the free surface is made to swell at the left wall of the vessel (see Fig. 2 (a)). High-power ultrasound, which can make the free surface scatter or spout, is then irradiated to the swollen free surface from the left side of the vessel, which would induce a circulation flow in the liquid as shown in Fig. 2 (b).

\subsection{Control of sound emitting position with ar- rayed source}

In a chemical analyzer that must process a variety

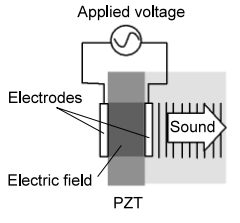

(a) PZT applied voltage

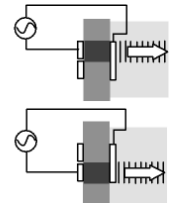

(b) Principle of selecting the position of ultrasound
Fig. 3 Schematic of an arrayed sound source

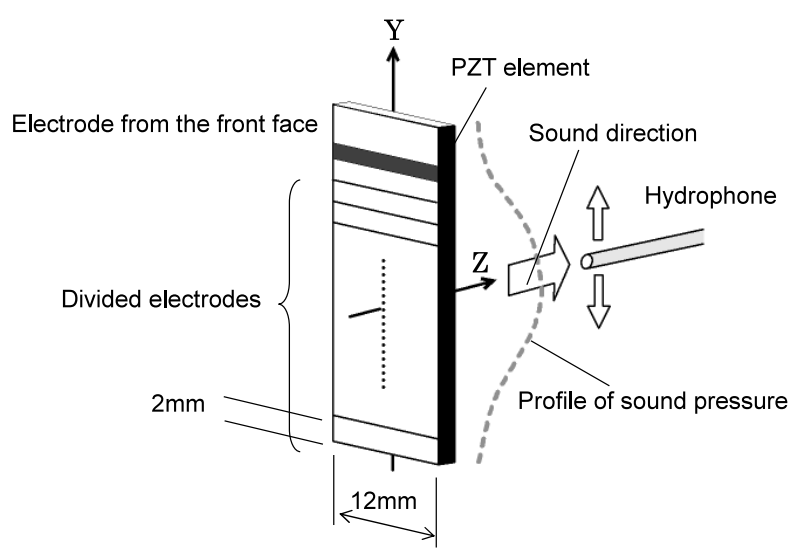

Fig. 4 Test sample of an arrayed sound source

of tests, the volume of the samples and reagents vary according to each test. In the non-contact mixing method in Fig. 2 (b), the position of the side sound source must be adjusted after the volume changes. Moving the sound source is one means of adjusting the emitting position. However, we simplified the means of the adjustment by using an array method that controls the emitting position without any moving elements.

The arrayed sound source we developed is based on the following principle. Generally, a piezoelectric element is equipped with electrodes shown in Fig. 3 (a), and applying a sinusoidal voltage to the electrodes makes the piezoelectric element generate sound. If the electrode is divided into several segments, by selecting the segments to which voltage is applied, the emitting positions can be selected of several possible settings as Fig. 3 (b) show. We conducted experiments to confirm whether the arrayed sound sources could be made to work like this. The test piece of the arrayed sound source we used in the experiment is in Fig. 4. It has a 12-mm-wide electrode divided into segments with a 2-mm pitch. The sound field (pressure distribution) irradiated from the arrayed sound source was measured with a hydrophone (NTR Systems Corp. TNU100A).

To confirm whether the sound emitting position had changed, three different patterns of sound field from the arrayed sound source were measured. In all three cases, a set of three contiguous segments (6-mm pitch) $12-\mathrm{mm}$ apart was selected. The measured profiles of the sound field are in Fig. 5(a). It is clear that the profile changes 


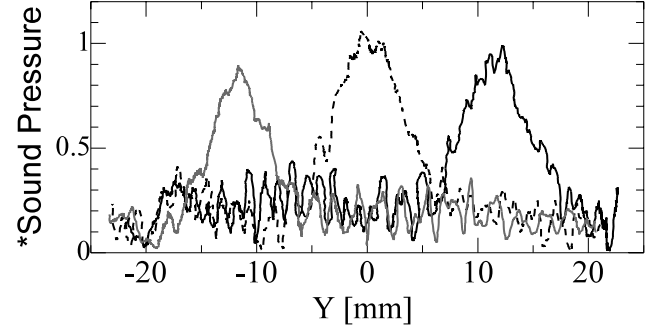

(a) Measured profile of sound field

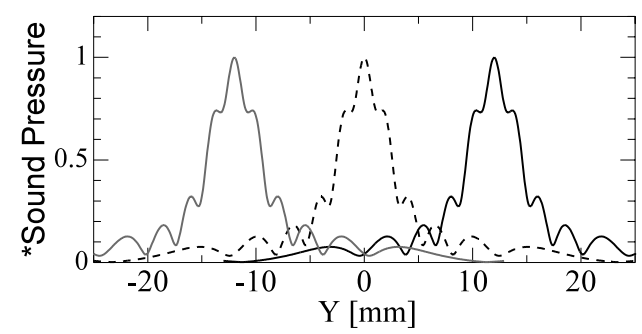

(b) Theoretical profile of sound field

Fig. 5 Comparison between measured and calculated sound fields ( $*$ Normalized by maximum pressure)

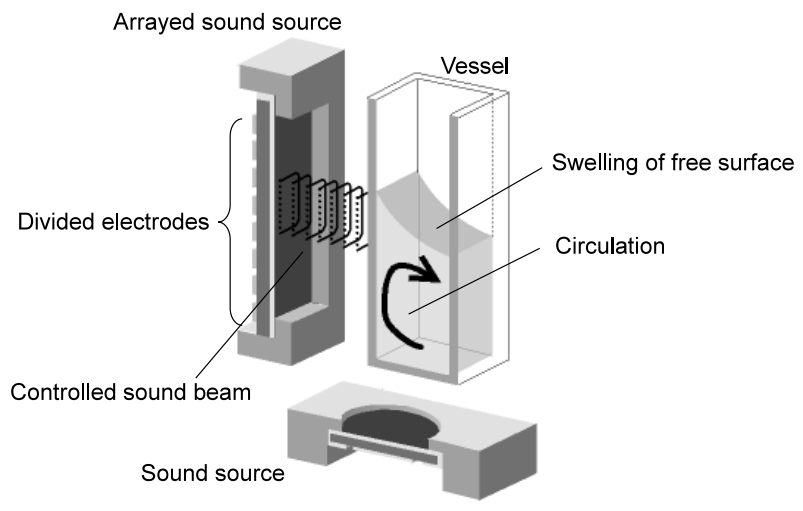

Fig. 6 Schematic diagram of proposed mixing device

according to the voltage applied to the three sets of three consecutive segments. To validate these sound profiles, we did simulation based on acoustic theory ${ }^{(6)}$ for a similar sound field to the measured one. The simulated profiles are in Fig. 5 (b). Figure 5 (a) and (b) reveal that the arrayed sound source performed as expected.

Screen-printing was used to fabricate the arrayed electrode pattern to an accuracy of about $10 \mu \mathrm{m}$. This is suitable for mass production and is very inexpensive. Utilizing these unique advantages, we produced the arrayed sound source by dividing one side of the electrode into several segments and adjusted the emitting position without using any moving elements.

\subsection{Design of non-contact mixing device}

Figure 6 is a schematic diagram of the designed mixing device. It consists of a vessel and two types of sound sources, both of which are made from piezoelectric elements (PZT: Lead Zirconate Titanate). The piezoelectric element whose electrode was arrayed into seg-

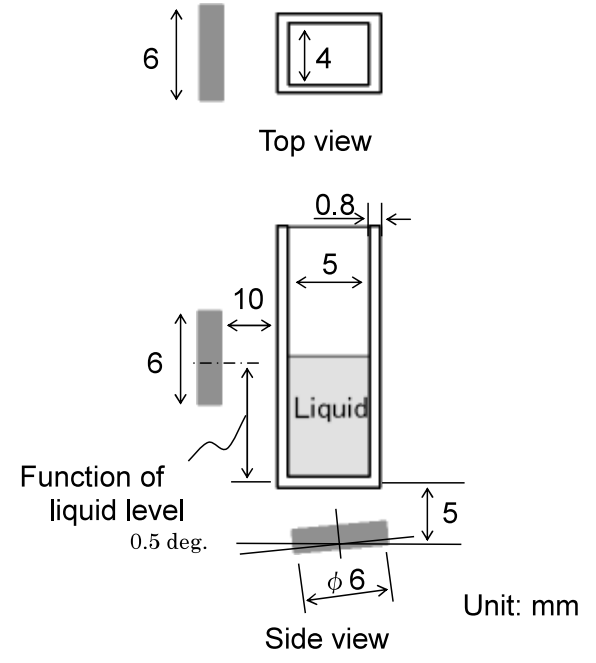

Fig. 7 Size and arrangement of sound sources

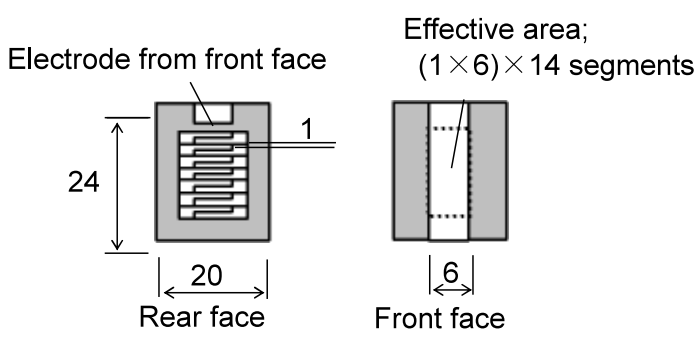

(a) For side sound source

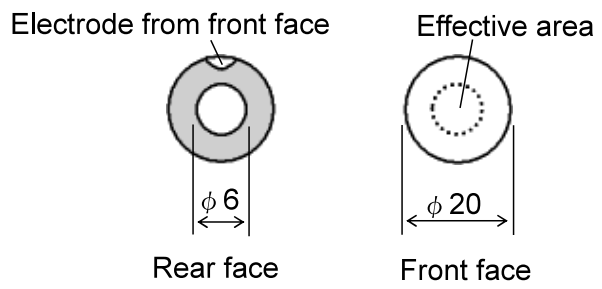

(b) For downward sound source

Fig. 8 Designed pattern of PZT electrode

ments was installed on the side of the vessel. The another piezoelectric element whose electrode was not divided was installed beneath the vessel. Although not shown in Fig. 6, the vessel and the sound sources were placed in water, thereby the sounds propagate thorough the water from the sound source to the vessel. A 0.8$\mathrm{mm}$ thick, $4 \times 5-\mathrm{mm}$ cross-sectional area (on the inside) of a vessel made from resin was used. The size and the disposition of the sound sources were in accordance to Fig. 7. Figure 7 (a) shows the side sound source (arrayed sound source), whose cross-sectional area is $6 \times 6$ $\mathrm{mm}$ square. Figure 7 (b) shows the bottom sound source, whose cross-sectional area is a circle having $6 \mathrm{~mm}$ in diameter. The shapes of the electrodes for the side and bottom sound sources were designed according to Fig. 8 (a) and (b) show, respectively. The area in which both sides of the electrodes overlap is the effective vibrating part of the piezoelectric element, and the areas on the side and 
bottom sound sources are indicated in Fig. 8 (a) and (b) by the dotted lines. In Fig. 8 (a), the electrode of the side sound source is divided into 14 segments (one segment is 6-mm wide with a 1-mm pitch). Therefore, by applying voltage to six contiguous segments selected from those 14 segments, the position of the emitting sound can be adjusted in units with a pitch of 1-mm.

To determine the proper level of voltage to apply to each piezoelectric element, we established the relationship between voltage and the swelling height of the free surface. Figure 9 is the schematic of the apparatus which is used for investigating the relationship between the voltage applied to the piezoelectric element and the swelling height of the free surface. The sound source was placed in the water tank at a $10-\mathrm{mm}$ depth from the free surface, and the relationship between the applied voltage and swelling height was investigated. We did the following tests to simulate the attenuation of sound at the vessel wall. The test piece was made of the same material and had the same thickness as the vessel wall was installed between the sound source and the free surface (5-mm bellow free surface) as Fig. 9 shows. The swelling height was measured by touching the free surface with a needle connected to a Z-stage. The height measurements are in Fig. 10, which shows that a 6-mm-diameter electrode (bottom sound source) and a $6 \times 6$-mm-square electrode

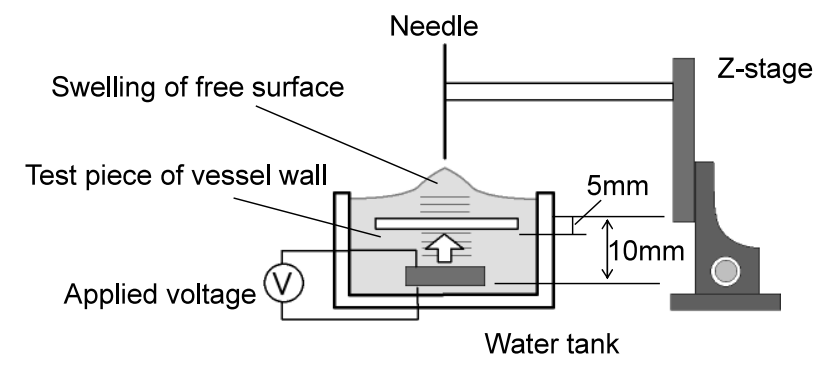

Fig. 9 Evaluation method for the threshold between swelling and scattering

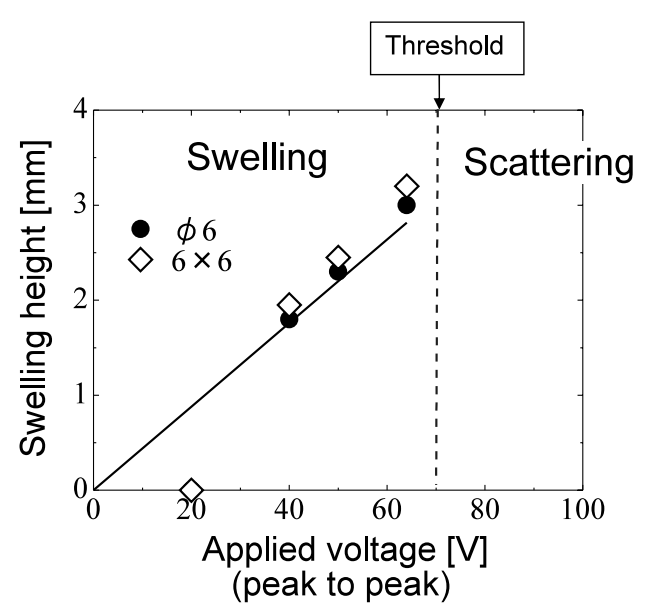

Fig. 10 Relationship between applied voltage to PZT and swelling height of free surface (side sound source) yielded almost the same results. When a voltage of $20 \mathrm{~V}$ (peak-to-peak, hereafter referred to as Vpp) was applied, the free surface only vibrated slightly, and no clear swelling was visible. When the applied voltage was increased from $40 \mathrm{Vpp}$ to $60 \mathrm{Vpp}$, clear swelling appeared. When the applied voltage exceeded $70 \mathrm{Vpp}$, there was scattering from the free surface. These results indicate that the threshold between the swelling and scattering of these piezoelectric elements is $70 \mathrm{Vpp}$. From these results, we determined that a voltage of $60 \mathrm{Vpp}$ is applied to the bottom sound source for the swelling the free surface, and $100 \mathrm{Vpp}$ to the side sound source for spouting are applied in our mixing device.

\section{Performance Evaluation}

\subsection{Flow visualization}

The mixing flow in our device was visualized with a dye (Amido black). Figure 11 has typical flow mixing of $156-\mu \mathrm{L}$ water and $4-\mu \mathrm{L}$ dye. The visualization captures the circulation of the liquid in the vessel and the water and dye was mixed in $1 \mathrm{sec}$. This visualization confirms the validity of our mixing method described in the section 2.1 and Fig. 2.

\subsection{Evaluation of mixing performance}

To evaluate the mixing performance more qualitatively, two points of laser-beam light were transmitted in to the water-dye mixture. Figure 12 is a schematic diagram of the apparatus used in this evaluation. Optical fibers (1-mm diameter) transmit the detected beam to photodetectors, which transform the beams into electrical signals. Figure 13 has typical waveforms for the outputs of the photodetectors. Note that ultrasound was emitted to the vessel for $0.5 \mathrm{sec}$. This suggests that the difference between two outputs after mixing exists, thereby the fluid in the vessel is not homogeneous. Obviously, if the fluid in the vessel is not homogeneous after mixing, the two transmitted light signals would be different. However, although there are no difference in output when the fluid is fully homogeneous, there is still the possibility that the fluid will be heterogeneous even if there are no differences in output. For example, when the mixing time is too short, the state of the fluid after mixing barely changes from the initial state when the dye is injected into the bottom of the vessel. The difference between the transmitted light outputs through the upper fluid in the vessel is almost zero. We thus define the efficiency of mixing as follows, using the two measured transmitted beams in the two steps explained below.

First, two transmitted light signals were measured when they passed through pure water (i.e. a "cell-blank" condition) and these signals were denoted as $P h_{10}$, and $P h_{20}$. The two transmitted beams measured after mixing the liquid and dye with the mixing device were denoted as $P h_{1 m}$ and $P h_{2 m}$, respectively. Then, the transmitted beams 


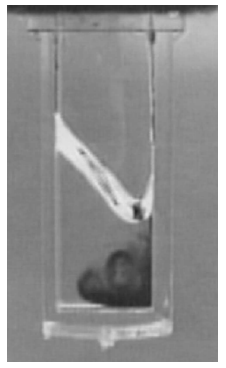

start

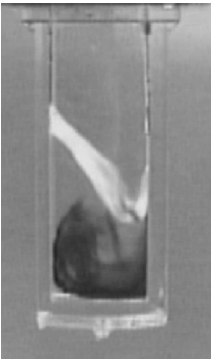

$0.3 \mathrm{sec}$. after
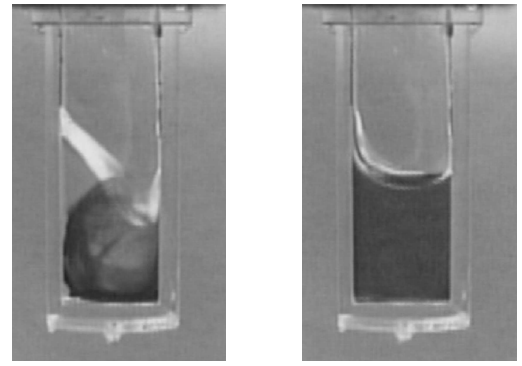

$0.6 \mathrm{sec}$. after $\quad 1.0 \mathrm{sec}$. after(stop)

Fig. 11 Visual demonstration of non-contact mixing method

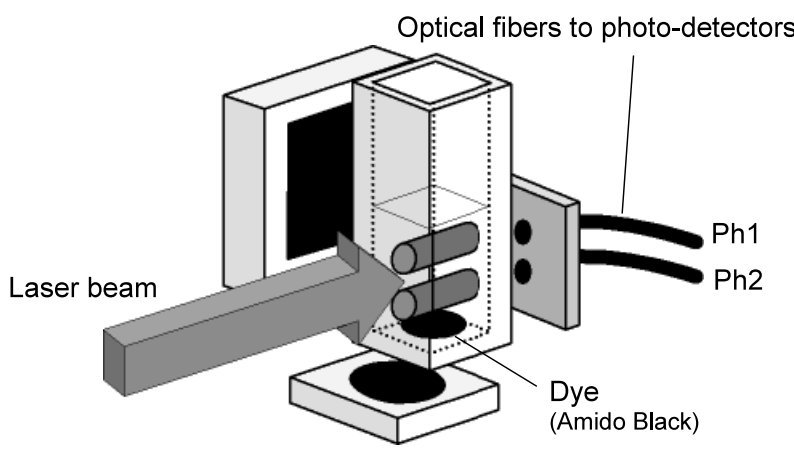

Fig. 12 Evaluation system for mixing performance

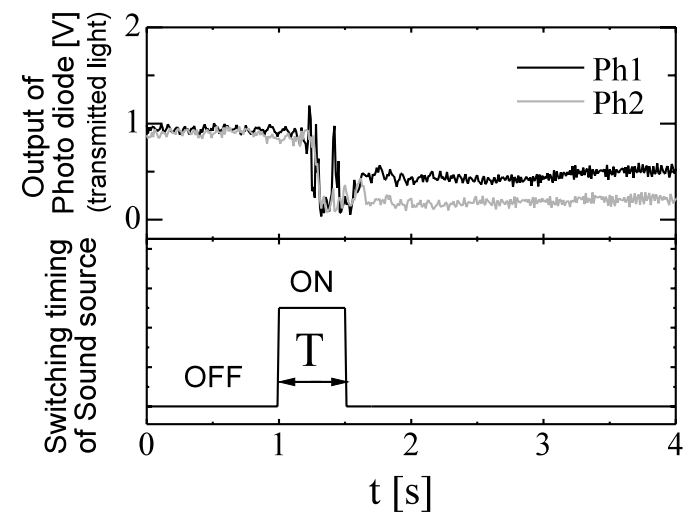

Fig. 13 Typical waveform of output from photo-detector

measured after full mixing was accomplished with other mixing methods were denoted as $P h_{1 s}$ and $P h_{2 s}$. To reduce the effects of electrical noise, we used a 0.5-s time averaging in all measurements.

Second, signals $P h_{10}, P h_{20}, P h_{1 m}, P h_{2 m}, P h_{1 s}$, and $P h_{2 s}$, were used to calculate absorbencies as follows,

$$
\begin{aligned}
& A_{\text {im }}=-\log \left(P h_{i m} / P h_{i 0}\right) \\
& A_{i s}=-\log \left(P h_{i s} / P h_{i 0}\right)
\end{aligned}
$$

where $i=1,2$. Equation (1) gives us the absorbance after mixing with our new mixing method, and Eq. (2) gives us that after mixing by the standard method. Deviation in these absorbencies is defined by the following equation which was used as a measure of mixing efficiency,

$$
P=1-\frac{1}{2} \sum_{i=1}^{2} \frac{\left|A_{i m}-A_{i s}\right|}{A_{i s}}
$$

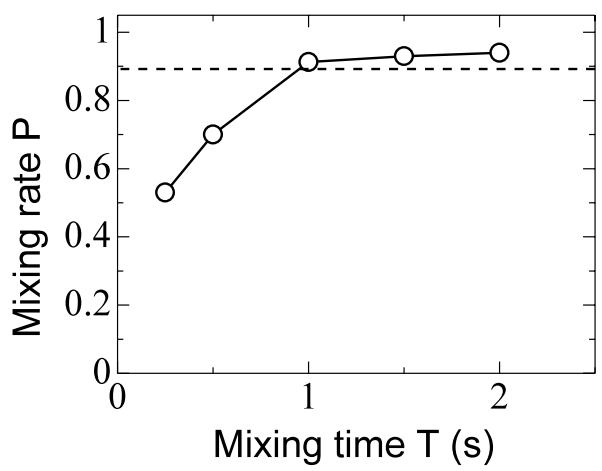

(a) Mixing time versus mixing rate

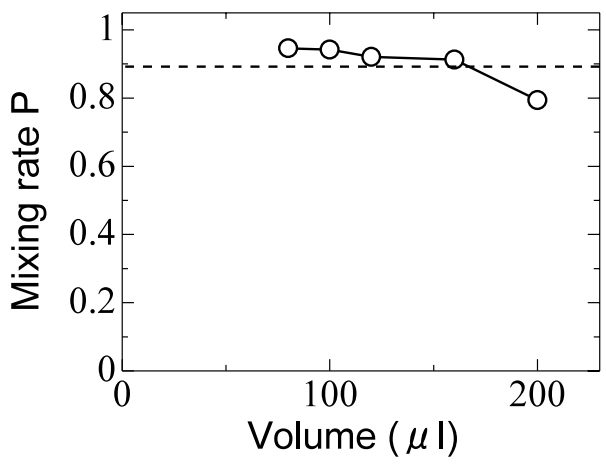

(b) Liquid volume versus mixing rate

Fig. 14 Evaluation results for mixing performance

We called the value derived "Mixing rate $P$ " from Eq. (3). This value is nearly 1.0 when the liquid is fully mixed, but nearly zero when it is incomplete. Taking the background noise of the evaluation system into account, we assessed that the liquid became homogeneous when the mixing rate was above 0.9 .

We then investigated dependence of the mixing time on the mixing rate. Figure 14 (a) shows the results when $156 \mu \mathrm{L}$ of water and $4 \mu \mathrm{L}$ of dye were mixed. This figure suggests that a mixing time of at least $1 \mathrm{sec}$. is needed to obtain a mixing rate of 0.9 in this case. Next, the mixing rate $P$ was evaluated in order to evaluate the dependence of the volume on mixing rate $P$, varying the liquid volume in the vessel. Figure 14 (b) shows the relationship of liquid volume with the mixing rate $P$ measured under the 
condition that the mixing duration is fixed to $1.0 \mathrm{sec}$. Taking the threshold 0.9 of the mixing rate into account, the mixing method can mix up to $160-\mu \mathrm{L}$-volume of the liquid. From these results, it is demonstrated that our new mixing method can mix the $160-\mu \mathrm{L}$-volume of the liquid at least in $1 \mathrm{sec}$.

\section{Conclusion}

We proposed a new mixing method of applying an acoustic radiation pressure generated at a free surface to induce flow without physical contact to the liquid, and verified the approach experimentally. In the present study, the following conclusions were obtained.

(1) Ultrasound has to be irradiated to the free surface in this method. Therefore, there was a need to adjust the position of ultrasound irradiation when the volume of the liquid to be mixed is changed. For this, we develop an arrayed sound source, whose side electrode is divided into several segments. By applying the voltage to proper electrode segments, the position of ultrasound irradiation can be moved without actually using a moving element. This arrayed sound source brings about several merits (simplicity, high reliability and low cost) to the mixing device configuration.
(2) Evaluated mixing performance show that $80-$ $160 \mu \mathrm{L}$-volume of water and some micro litter of dye in a vessel (cubic shaped vessel made from a resin, $4 \times 5-\mathrm{mm}$ cross sectional area, $0.8-\mathrm{mm}$ thick wall) can be mixed at least in $1 \mathrm{sec}$.

\section{References}

( 1 ) Yang, Z., Goto, H., Matsumoto, M. and Maeda, R., Ultrasonic Micromixer for Microfluidic Systems, MEMS2000, (2000), pp.668-673.

( 2 ) Vivek, V., Zeng, Y. and Kim, E.S., Novel AcousticWave Micromixer, MEMS2000, (2000), pp.80-85.

(3) Wang, H. and Kim, E.S., Ejection Characteristics of Micromachined Acoustic-Wave Liquid Ejector, Transducers '99, (1999), pp.1784-1787.

( 4 ) Andreev, A.M. and Ginzburg, V.M., Dynamic Liquid Surface Measurements by Talbot Effect, Proc. SPIE Int. Soc. Opt. Eng., (1997), pp.280-284.

( 5 ) Cinbis, C., Mansour, N.N. and Khuri-Yakub, B.T., Effect of Surface Tension on the Acoustic Radiation Pressure-Induced Motion of the Water-Air Interface, J. Acoust. Soc. Am., Vol.94, No.4 (1993), pp.2365-2372.

( 6 ) Junger, M.C. and Feit, D., Sound, Structures, and Their Interaction (Second Edition), (1986), pp.75-90, The MIT Press. 